\title{
SYNCHROTRON RF SYSTEM FOR THE JAERI-KEK JOINT PROJECT
}

\author{
C. Ohmori, E. Ezura, Y. Hashimoto, Y. Mori, A. Takagi, T. Uesugi, M. Yoshii, \\ KEK, 1-1 Oho, Tsukuba, 305-0801, Japan \\ F. Tamura and M. Yamamoto, JAERI-Tokai, Tokai, Japan
}

\begin{abstract}
A synchrotron $\mathrm{rf}$ system has been designed for the JAERI-KEK Joint Project for High-Intensity Proton Accelerators in Japan. A new type of rf cavity loaded with MA(Magnetic Alloy) cores will be used for synchrotrons in order to achieve the high field gradient for the rf acceleration. The status of $R \& D$ on the cavity is also reported.
\end{abstract}

\section{INTRODUCTION}

The accelerator complex of the JAERI-KEK Joint Project[1,2] consists of a $400 \mathrm{MeV}$ linac, $3 \mathrm{GeV}$ and 50 $\mathrm{GeV}$ Synchrotrons. The accelerators provide high intensity proton beams for high-energy physics, solid state physics, biology and other fields. The accelerators will be constructed in the JAERI-Tokai site. The $3 \mathrm{GeV}$ Synchrotron is a rapid-cycling ring and provides $1 \mathrm{MW}$ proton beams for a pulsed neutron source and $15.6 \mu \mathrm{A}$ for the $50 \mathrm{GeV}$ PS, respectively. The main parameters of the synchrotrons are listed in Table 1.

Table 1: Main parameters

\begin{tabular}{|l|c|c|}
\hline & 3GeV PS & 50GeV PS \\
\hline Rep. Rate & $25 \mathrm{~Hz}$ & $0.3 \mathrm{~Hz}(3.42 \mathrm{~s})$ \\
\hline Rise time & $20 \mathrm{~ms}$ & $1.9 \mathrm{~s}$ \\
\hline Harmonics & 2 & 10 \\
\hline Circumference & $313.5 \mathrm{~m}$ & $1567.5 \mathrm{~m}$ \\
\hline Energy & $0.4-3 \mathrm{GeV}$ & $3-50 \mathrm{GeV}$ \\
\hline rf frequency & $1.36-1.86 \mathrm{MHz}$ & $1.86-1.91 \mathrm{MHz}$ \\
\hline Max. Voltage & $420 \mathrm{kV}$ & $280 \mathrm{kV}$ \\
\hline Max. $\phi \mathrm{s}$ & 48 degree & 28 degree \\
\hline Max. $\Delta \mathrm{p} / \mathrm{p}$ & $1 \%$ & $0.7 \%$ \\
\hline
\end{tabular}

\section{RF SYSTEM}

The $50 \mathrm{GeV}$ Synchrotron will accumulate 8 bunches by the bunch-to-bucket transfer from the $3 \mathrm{GeV}$ Synchrotron. The $3 \mathrm{GeV}$ Synchrotron will cycle four times for each accumulation period of the $50 \mathrm{GeV}$ Synchrotron. The rf will operate at the 10th harmonics of the revolution frequency and two buckets are empty and reserved for the rise time of the kicker magnets to extract the beam at the top energy.

The rf system has been designed to accelerate $4.12 \mathrm{X}$ $10^{13}$ protons per bunch. The beam intensity is high enough to affect the rf system as the beam loading effects and to cause the significant beam loss by instabilities. Stability of the rf system for both beam loading and instabilities is the most significant to design the system. It is also required to supply the large rf power for the acceleration. It is $1.72 \mathrm{MW}$ for the $50 \mathrm{GeV}$ Synchrotron and the power per cavity is $288 \mathrm{~kW}$. In order to supply this rf power, high power tube is necessary.

\subsection{MA Cavity}

Magnetic Alloy-loaded cavity[3,4] will be used for both synchrotrons. The MA has a very high permeability, low $Q$ value and high impedance. One of the interesting characteristics is that the core is completely stable for the rf magnetic field. Because of high Curie temperature of the core, it is also stable for the temperature change. The severe temperature control which is required for ferrite loaded cavity is not necessary for this cavity. These characteristics can realise a high gradient acceleration system.

The $\mathrm{Q}$ value of the cavity can be increased by a cutcore configuration. The advantage of the scheme is that the shunt impedance is constant for the wide range of $\mathrm{Q}$ value (1-20). The optimum $Q$ value is between 10 and 20 for the $50 \mathrm{GeV}$ Synchrotron to reduce the transient beam loading and bucket distortion effects, and to sweep the rf frequency without any tuning. The tuning loop is not necessary for MA cavity if the Q value is lower than 20 to cover the rf frequency. The removal of the tuning system makes the rf control system simple and enlarges the stability of the system. For the $3 \mathrm{GeV}$ Synchrotron, the optimum Q value is 2-3.

\subsection{Beam Loading}

In order to avoid Robinson type instabilities without using rf feedback, the amount of beam power must be less than the power dissipation in the cavity. The cavity impedance was chosen to be about $1 \mathrm{k} \Omega$ per gap. The peak power consumption in the cavities and beam power are 2.2 MW and 1.7 MW, respectively, for the $50 \mathrm{GeV}$ Synchrotron. With concerning the tube resistance, the relative loading parameter, $\mathrm{Y}=\mathrm{IB} / \mathrm{I} 0$, is almost 1 . Figure 1 shows that phaser diagram for the whole machine cycle. The power load sawn by the amplifier is close to the pure resistance that is an optimum load for the tetrode. For the $3 \mathrm{GeV}$ Synchrotron, the cavity impedance is $700 \Omega$. The relative loading parameter is less than 1 except for the extraction period. A feed forward beam compensation and counter phasing are necessary to reduce the rf voltage for the beam emittance matching.

Another compensation system will be required to suppress the periodic transient effects while the injection and acceleration for the $50 \mathrm{GeV}$ Synchrotron because the empty buckets exist in the ring. The most severe transient effects occur when the 6 buckets are filled and 4 are empty during the injection process. The rf system is designed to compensate the effects. The bandwidth of the 
system is enough wide and the high power amplifier will supply the extra power for this purpose. When loaded Q of the system is 10 , the major components of wake field on the cavity induced by the beam is $\mathrm{H}=9,10$ and 11 . The rf system will have wide bandwidth to compensate these three harmonic components and $20 \%$ higher feed forward current will be required to cancel all. The bucket distortion is another problem to consider because the bunching factor of the synchrotron will be 0.05 and peak current be $180 \mathrm{~A}$ at the flat top. In case of $\mathrm{Q}=10$, the wake voltage is not negligible, however the bucket distortion is marginal.

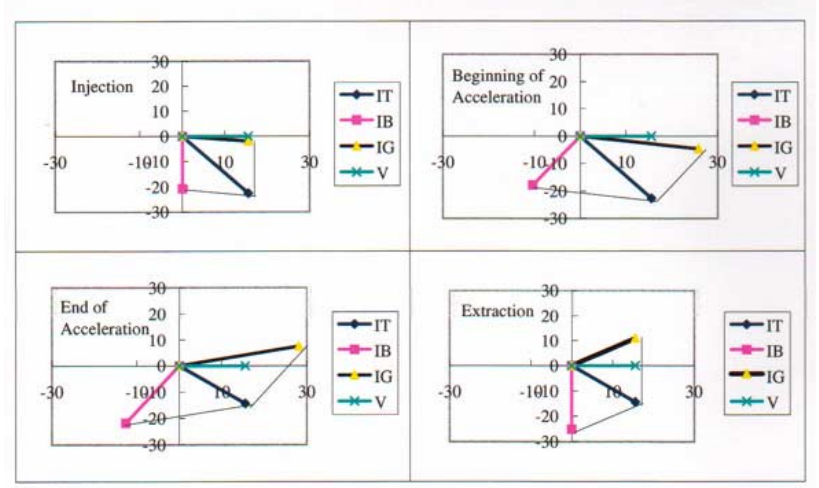

Fig. 1. Phase diagram for the $50 \mathrm{GeV}$ Synchrotron. By choosing the optimum $\mathrm{Q}$ value and resonant frequency, the cavity and beam load will be close to a pure resistive one through the whole machine cycle. The relative loading parameter is almost 1 in this case.

\subsection{Cavity Design}

The power consumption in the cores is given by using the gap voltage $(15.5 \mathrm{kV})$, impedance $(1 \mathrm{k} \Omega)$, number of cores (8 per gap), and duty factor $(60 \%)$. The peak power per gap is $122 \mathrm{~kW}$ and average power of $9 \mathrm{~kW}$ will be dissipated in a core. The power density is $0.78 \mathrm{~W} / \mathrm{cc}$. For $3 \mathrm{GeV}$ Synchrotron, it is $0.5 \mathrm{~W} / \mathrm{cc}$

There are two different schemes to cool the cores. One is, so called, direct cooling [5,6] and the other is indirect one. In case of direct cooling, the cores are stored in the water tank and cooled by the water directly. One side of the water tank is made by the FRP plate to obtain the high voltage at the gap. The cooling method is very powerful and efficient. However, because of the large dielectric constant of the water, about 80 , the large capacitance exists in the cavity. The cavity can not resonate at the frequency higher than $5 \mathrm{MHz}$. And some part of the impedance will be significantly lost at the high frequency. For the purpose of the $3 \mathrm{GeV}$ and $50 \mathrm{GeV}$ Synchrotrons, the method is still possible to use because the $\mathrm{rf}$ frequency is 1.3 to $4 \mathrm{MHz}$ although the impedance is reduced by $20 \%$. The mechanical configuration is another problem for this method to support the cores in the water tanks when it is necessary to change the $Q$ value. And, the quality of the water will affect the cavity impedance

As an alternative, the indirect cooling will be used for the project. A good insulation material should be installed between the cores and cooling plate to avoid the impedance reduction by the capacitance effects. To reduce the capacitance between the cores and metal plate, the thickness should be larger than a few $\mathrm{mm}$ as shown in Fig. 2. However, the cooling of the core will be another problem. The mixture of the polyimide and Aluminium Nitride will give us a good heat transfer and high voltage insulation. The sample of this mixture shows the excellent heat conductance of $1.6-5 \mathrm{~W} / \mathrm{mK}$ and it is one order better than ordinary epoxy $(0.2 \mathrm{~W} / \mathrm{mK})$ and plastics. The polyimide can be used at the very high temperature because the dissolve temperature is 340 degree C. Assuming the $12 \mathrm{~kW}$ is dissipated in the core, uniformly, the maximum temperature in the cores is about 80 degree $\mathrm{C}$ when we use the insulator of $1.6 \mathrm{~W} / \mathrm{mK}$. As shown in Fig.2, three cores are cooled from both surfaces. However, one core which will be located near the acceleration gap will be cooled from one surface in order to avoid the reduction of the impedance.

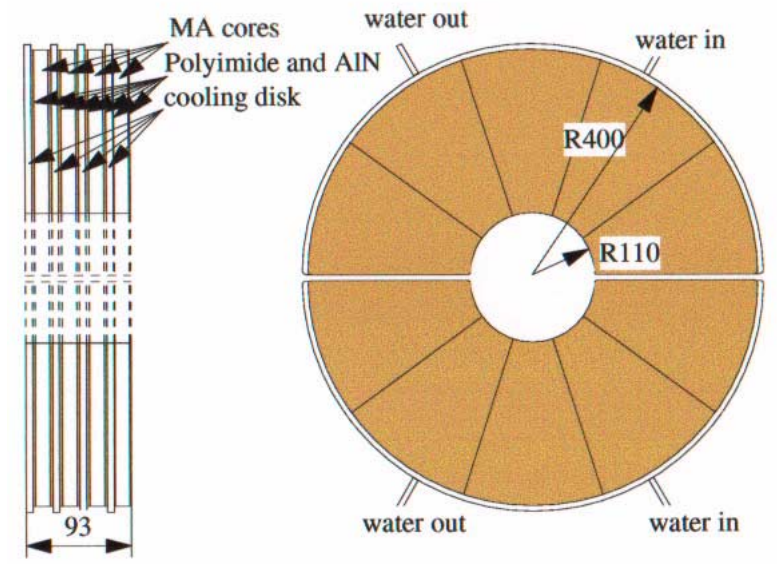

Fig.2. A stack of MA cores and cooling disks.

The cavity for the joint project has 3 gaps and each gap consists of 2 set of quarter-waver structure using 4 MA cores as shown in Fig. 3. The size of core is 80cm O.D. X $24 \mathrm{~cm}$ I.D. X $2.5 \mathrm{~cm}$ thickness. The MA cores are cut in half and separated by about $1 \mathrm{~cm}$. The cores are covered by $0.5 \mathrm{~cm}$ thick polyimide resin and cooled by $1.2-\mathrm{cm}$ thick water cooled copper heat sinks. The polyimide resin contains Aluminium-Nitride powder with high thermal conductivity by $70 \%$ in weight. The polyimide resin reduces the coupling capacitance between the heat sink and cores which may reduce the cavity impedance. The power loss in a core is less than $10 \mathrm{~kW}$ and the water flow per heat sink is 5 litter per minutes. Four sets of $400 \mathrm{pF}$ capacitor will be used to resonate at $2 \mathrm{MHz}$. Loaded and unloaded Q values of the cavity are 10 and 16 , respectively. The shunt impedance is $1 \mathrm{k} \Omega$ per gap. Two gap short relays are used for a cavity gap to avoid the any instability while the rf power is turned off. Two sets of gap voltage monitor are installed in a gap of the cavity.

The cavity will be powered by a $1 \mathrm{MW}$ final amplifier. The cavity voltage is $50 \mathrm{kV}$ at 3.4-second repetition time with a $60 \%$ duty cycle. 


\subsection{Cavity $R \& D$}

The indirect cooling method has been tested using a one gap cavity as shown in Fig. 3. In the cavity, 3 small MA cores are loaded. The cores are cooled by the aluminium cooling disk indirectly. Between cores and cooling disk, the polyimide insulators and inorganic glue are inserted. The thickness of the insulators is $5 \mathrm{~mm}$ and the thermal conductance is $1.6 \mathrm{~W} / \mathrm{mK}$. The inorganic glue also has a good conductance of $2 \mathrm{~W} / \mathrm{mK}$.

The cavity impedance has been measured as shown in Fig. 4. At $4 \mathrm{MHz}$, a MA core has an impedance of about $150 \Omega$. The cavity impedance was $380 \Omega$ at $4 \mathrm{MHz}$ and $480 \Omega$ at $8 \mathrm{MHz}$, respectively. As shown in Fig. 5, only $15 \%$ of the impedance is lost by the effects caused by the capacitance between core and cooling disk. If the insulators were not installed, the impedance will be less than a half.

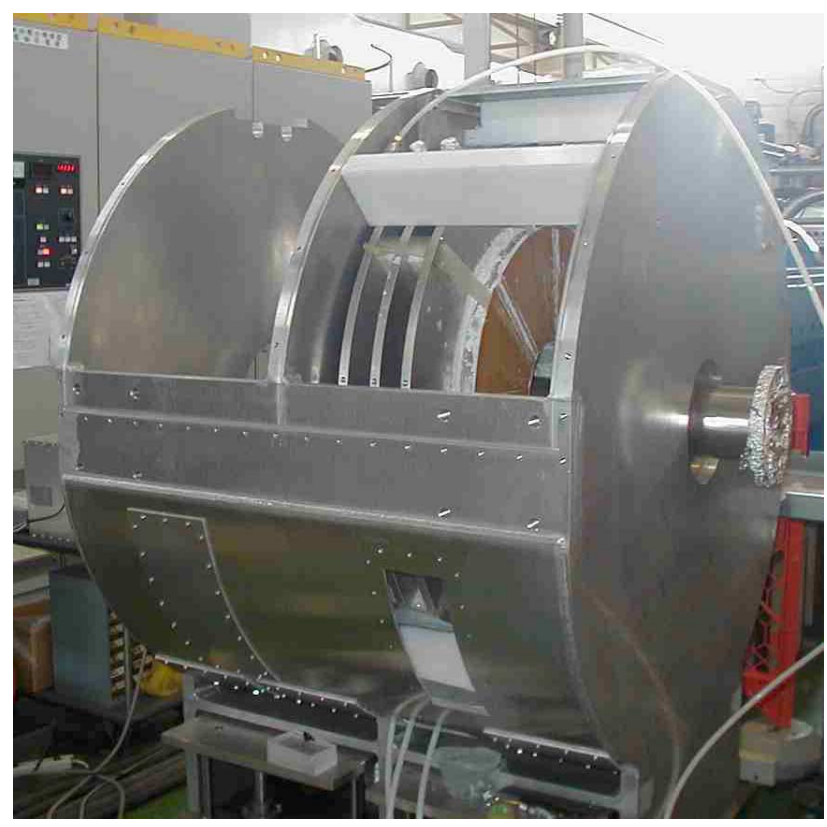

Fig. 3. A test cavity using an indirect-cooling method.

Power test using a test cavity has been performed. The test cavity has achieved $4.2 \mathrm{kV}$ with a $50 \%$ duty cycle. An average power of $4 \mathrm{~kW}$ per core has been dissipated. As a power density, it will be $0.52 \mathrm{~W} / \mathrm{cc}$ and the goals are $0.5 \mathrm{~W} / \mathrm{cc}$ for the $3 \mathrm{GeV}$ ring and $0.9 \mathrm{~W} / \mathrm{cc}$ for the $50 \mathrm{GeV}$ Synchrotron. The surface temperature of the core that was cooled by only one cooling disk as shown in Fig. 3 has been measured. Some parts of the core, especially the outside edge, has not been cooled well because there were voids in the inorganic glue. Other parts, including inside edges of the core where it is expected to be the hottest spot, have been cooled, marginally.

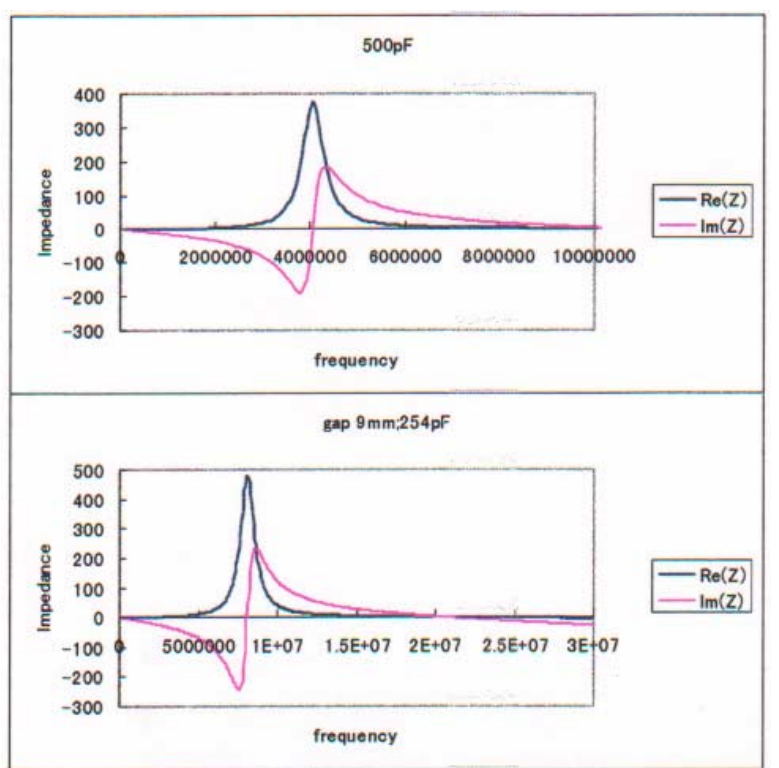

Fig. 4. Impedance of the test cavity. Because of the insulator between cores and cooling disk, only $15 \%$ of the impedance is lost.

Recently, it is found that the core and cooling disks can be assembled without using inorganic glue. The so-called B-stage of the polyimide resin can be used to bind the cores and cooling disks.

\section{CONCLUSIONS}

A synchrotron rf system has been designed for the JAERI-KEK Joint Project. A new type of the cavity will be used to achieve the high filed gradient. A new cooling method has been developed.

\section{REFERENCES}

[1] The Joint Project team of JAERI and KEK, KEKReport 99-4, JAERI-Tech 99-056.

[2] Y. Yamazaki et al., "Accelerator complex for the Joint Project of KEK/JHF and JAERI/NSP", PAC99, New York, April 1999, p513.

[3] T. Uesugi et al., "New magnetic material for proton synchrotron rf cavity", Proc. of Symp. on Acc. Sci. and Tech., Nishiharima, Japan 1997.

[4] Y. Mori et al., "A new type of rf cavity for high intensity proton synchrotron using high permeability magnetic alloy", EPAC 1998, p299.

[5] C. Ohmori et al., "High Field Gradient Cavities Loaded with Magnetic Alloys for Synchrotrons", PAC99, New York, April 1999, p413.

[6] C. Ohmori et al., "RF acceleration systems for the joint project", EPAC2000, p1984. 